\title{
The Diagnostic Value of Magnetic Resonance Imaging in Identifying Unsuspected Malignancy in Patients Undergoing Percutaneous Vertebral Augmentation for Vertebral Compression Fractures
}

\author{
CHEW ZHIHONG, MD, MRCS, ${ }^{1}$ CHENG SHENG DA JOWELL, BENG ${ }^{2}$ SYED AFTAB, MD,${ }^{3}$ SEANG BENG \\ TAN, MBBS, MMED, ${ }^{1}$ CHANG MING GUO, MBBS, MMED (SURG), ${ }_{1}^{1}$ JOHN CHEN LI TAT, MBBS (NUI), \\ BAO,${ }^{1}$ POH LING FONG, MBBS (SPORE), MMED (ORTHO) ${ }^{1}$ WILLIAM YEO, BAPPSC, MPHYT, ${ }^{1}$ \\ MASHFIQUL A. SIDDIQUI, MBBS, MRCS (SURG), MMED (ORTHO) ${ }^{1}$ \\ ${ }^{I}$ Department of Orthopaedic Surgery, Singapore General Hospital, Singapore, ${ }^{2}$ Duke-NUS Medical School, Singapore, ${ }^{3}$ Department of Diagnostic Radiology, \\ Singapore General Hospital, Singapore
}

\begin{abstract}
Background: The aim of this study was to identify the rate of unsuspected malignancy in vertebral compression fractures (VCFs) treated with percutaneous vertebral augmentation procedures (PVAPs).

Methods: From 2004-2015, 410 patients with VCFs underwent PVAPs with biopsy in a single tertiary hospital. All patients had preoperative magnetic resonance imaging (MRI) read by consultant radiologists and reviewed by the performing surgeon prior to PVAPs. All procedures were performed by fellowship-trained spine surgeons. A patient was considered to have an unsuspected malignancy if preoperative MRI was negative for malignancy but histology from the operative biopsy was positive.

Results: A total of 44 of 45 patients $(97.8 \%)$ were identified to have malignancy on preoperative MRI. One patient had a negative MRI but positive biopsy (myeloma). This patient also had a positive myeloma panel. A total of 41 of 44 patients with suspicious MRI preoperatively had a history of malignancy with histology consistent with metastatic spread from the known primary. Two patients had a new diagnosis of malignancy (1 breast carcinoma, 1 metastatic cancer likely of breast or gastrointestinal origin). Younger patients were more likely to have a VCF due to malignancy (odds ratio, 28.33 in age $<60$ years).

Conclusions: Almost $98 \%$ of patients with malignancy (44 of 45 patients) could be successfully identified with a preoperative MRI. The addition of a myeloma panel to MRI identified all patients with malignancies prior to PVAP in our study. We recommend MRI and myeloma panel for all patients with VCFs to be treated with PVAPs. For patients who undergo a PVAP, routine biopsy should be performed.
\end{abstract}

Lumbar Spine

\section{INTRODUCTION}

Vertebral compression fractures (VCFs) occur in up to $20 \%$ of postmenopausal women and can lead to chronic, decreased quality of life and increased mortality. ${ }^{1,2}$ Osteoporosis is presumed to be the main cause of VCF in most cases. Percutaneous vertebral augmentation procedures (PVAPs), such as percutaneous vertebroplasty and kyphoplasty, are minimally invasive treatment options in the management of VCF unresponsive to conservative options. A bone biopsy can be done safely during the procedure at different levels. ${ }^{3-5}$

Rarely, VCF may be secondary to malignancy rather than osteoporosis alone. ${ }^{6-9}$ In up to $20 \%$ of patients, lesions in the spine may represent the first manifestation of malignancy. ${ }^{8}$ Malignant and nonmalignant VCFs may have overlapping imaging characteristics resulting in potential difficulty in identifying malignancy-related fractures. ${ }^{10}$ There is sparse literature on the rate of unsuspected malignancy in VCF. Chou et $\mathrm{al}^{11}$ reported a $1.1 \%$ rate of unsuspected malignancy in VCFs. The most recent study by Hansen et $\mathrm{al}^{12}$ reported the rate of unsuspected malignancy in patients undergoing percutaneous vertebral augmentation to be as high as $4.9 \%$, where preoperative clinical examinations, magnetic resonance imaging (MRI), and blood tests were negative but intraoperative biopsy result was positive. This study suggests that as many as 1 in 20 patients with a 
VCF may be harboring a malignancy that had been missed by MRI and blood investigations.

The aim of our study was to identify the rate of unsuspected malignancy in VCF treated with vertebral augmentation and the accuracy of preoperative MRI in detecting pathologic VCF secondary to a malignancy. The study also investigated if age was a risk factor for pathologic VCF secondary to malignancy.

\section{METHODS}

In this retrospective cohort study, all patients who underwent a PVAP for VCF from May 2004 to November 2015 were identified from the spine registry of a tertiary hospital and considered for the study. Only patients with intraoperative biopsy were included in the study. Exclusion criteria were thus patients who did not have an intraoperative biopsy during the cementation procedure. Case notes, blood tests, MRI scans, and histology from the biopsy during cementation procedure were reviewed for all patients.

All included patients had a preoperative MRI prior to PVAP, performed using a Siemens Avanto 1.5T MRI Scanner. Sagittal and axial images were captured on T1, T2, T1 fat-suppressed before and after contrast (where applicable), and Short Tau inversion recovery sequences. Intravenous contrast was used at the discretion of radiologists with patient consent. All MRIs were reported by consultant radiologists and reviewed by the consultant spine surgeon prior to performing PVAP.

The procedure was performed with patients in prone position and using sterile techniques. Using fluoroscopy to determine the location of the pedicle, a small incision at the superior edge of the pedicle was made, and a Jamshidi needle was inserted via a transpedicle approach into the vertebral body. A 2$\mathrm{mm}$ guide pin was passed through the Jamshidi needle, and the needle was exchanged for an obturator followed by a working cannula. A bone biopsy was obtained by inserting and twisting an obturator while applying suction with a syringe. Biopsy specimens then underwent fixation in Lillie AAF (ethanol, acetic acid formaldehyde) and subsequently were decalcified in buffered formic acid before dehydration and embedding in paraffin. Histologic, 4-mm-thick, hematoxylin-eosin-stained tissue sections were evaluated morphologically. If malignancy was suspected, the morphologic examination was supplied by immunohistochemistry analysis, in which antibodies were
Table 1. Patient demographics.

\begin{tabular}{lc}
\hline Parameter & Value \\
\hline Mean age, y (range) & $74.3(26-96)$ \\
Sex, n (\%) & \\
Male & $91(22.2)$ \\
Female & $319(77.8)$ \\
Vertebral levels of cement augmentation, n (\%) & $\mathrm{N}=410$ \\
T5 & $2(0.5)$ \\
T6 & $4(1.0)$ \\
T7 & $7(1.7)$ \\
T8 & $4(1.0)$ \\
T9 & $4(1.0)$ \\
T10 & $13(3.2)$ \\
T11 & $21(5.1)$ \\
T12 & $69(16.8)$ \\
L1 & $125(30.4)$ \\
L2 & $66(16.1)$ \\
L3 & $52(12.8)$ \\
L4 & $27(6.7)$ \\
L5 & $11(2.7)$ \\
S1 & $1(0.2)$ \\
\hline
\end{tabular}

selected by the pathologist directed by the suspected kind of malignancy (for example cytokeratins 7 and 20 for metastatic carcinoma, CD138 for multiple myeloma, etc.).

A patient was regarded to have an unsuspected malignancy if preoperative clinical history physical examination and MRI scans were negative but intraoperative histology was positive for malignancy.

All patients who underwent bone biopsies during PVAPs were stratified into different age groups and analyzed for rates of malignancy detected on biopsy. The age groups were younger than 60 years; 60 to 70 years; 70 to 80 years; and older than 80 years. The odds ratios for VCFs due to malignancy were calculated using multinomial logistic regression, with the control group being those older than 80 years. All statistical analysis was carried out using IBM SPSS Statistics version 24.0.

\section{RESULTS}

There was a total of 736 patients who had a PVAP for VCF during the time period, of whom 326 patients did not have a biopsy intraoperatively. The remaining 410 patients with VCF undergoing PVAP with routine biopsy, comprising 169 vertebroplasties and 241 kyphoplasties, were included in the study. The mean age of the patients was 74.3 years (range, 26-96 years), with 91 men $(22.2 \%)$ and 319 women $(77.8 \%)$. Most of the vertebral fractures occurred at the thoracolumbar junction (Table 1).

A total of 45 of the 410 patients $(11 \%)$ were found to have malignancy on biopsy (Figure 1), and of these $45,44(97.8 \%)$ were identified to have a malignancy 

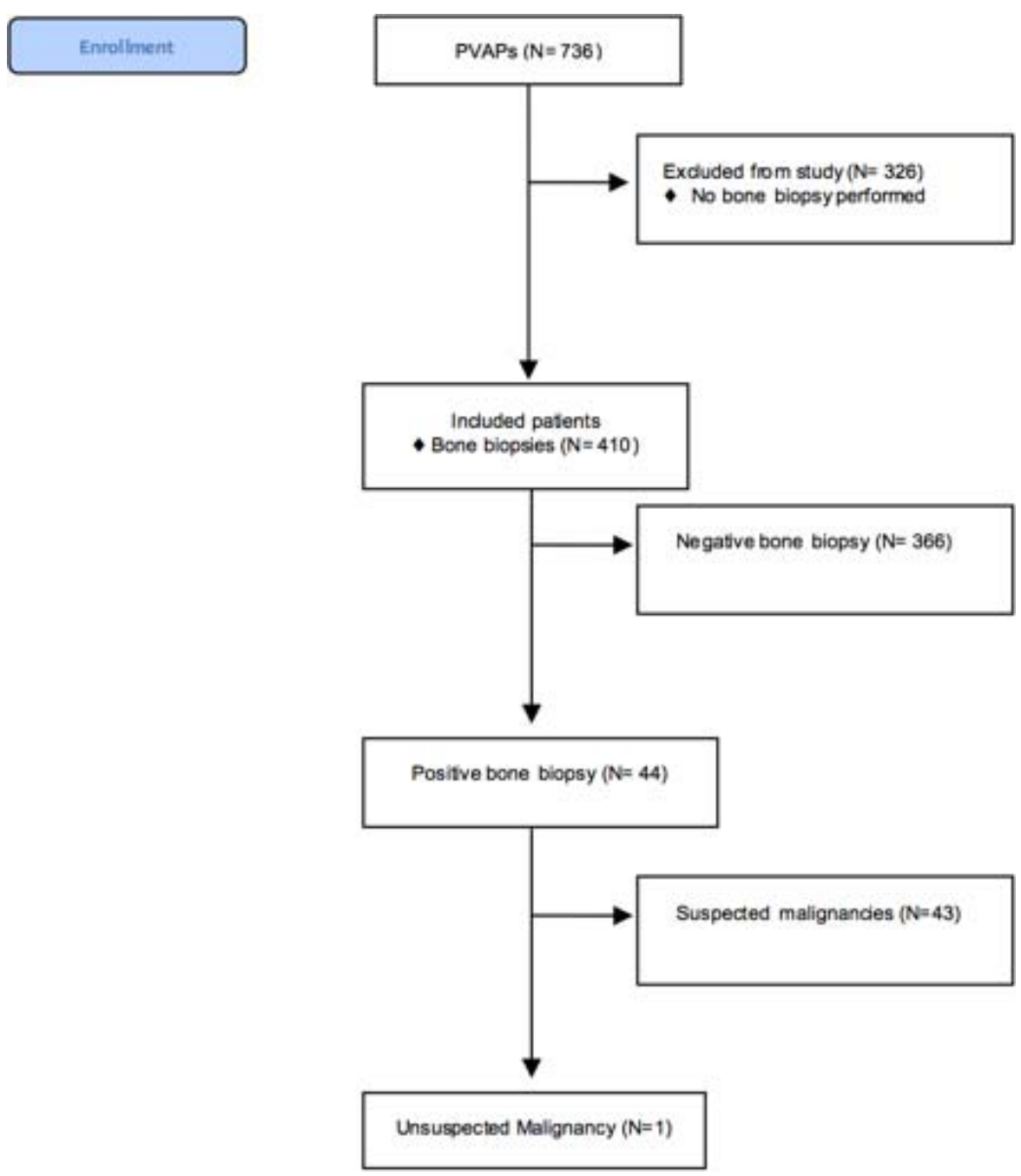

Figure 1. Flow chart of results.

on preoperative MRI. One patient had a negative MRI for malignancy but a positive operative biopsy for multiple myeloma. This patient also had positive findings of monoclonal band on blood tests. Table 2 reflects the histology of the patients.

Table 2. Biopsy results.

\begin{tabular}{lc}
\hline Positive Biopsies & No. $\mathbf{( \% )}, \mathbf{N}=\mathbf{4 5}$ \\
\hline Breast Ca & $15(33.3)$ \\
Lung Ca & $5(11.1)$ \\
Multiple myeloma & $5(11.1)$ \\
Colorectal Ca & $3(6.7)$ \\
Renal Cell Ca & $2(4.4)$ \\
Liver Ca & $2(4.4)$ \\
Pancreatic Ca & $2(4.4)$ \\
Gastric Ca & $2(4.4)$ \\
Lymphoma & $1(2.2)$ \\
Sigmoid Ca & $1(2.2)$ \\
Cervical Ca & $1(2.2)$ \\
Endometrial Ca & $1(2.2)$ \\
Esophagus Ca & $1(2.2)$ \\
Ca of unknown origin & $4(8.8)$ \\
\hline
\end{tabular}

Abbreviation: Ca, carcinoma.
Of the 44 patients who were suspected to have a malignancy on MRI preoperatively, 41 patients had a history of malignancy with histology being consistent with metastatic spread of the known malignancy. Two of the patients had newly diagnosed malignancies ( 1 breast carcinoma and another metastatic cancer likely of breast or gastrointestinal origin).

Younger patients were found to have a higher percentage of pathologic fracture compared with the older age groups. Rates of malignancy in the various age groups are shown in Figure 2. Odds ratios for VCFs due to malignancy were found to be 28.33 (95\% confidence interval [CI], 7.8-103.5), 12.59 (95\% CI, 4.8-33.3), and 2.52 (95\% CI, 1.15.7) for age groups younger than 60 years, 60 to 70 years, and 70 to 80 years, respectively, compared with patients who are age 80 years (Table 3 ). 


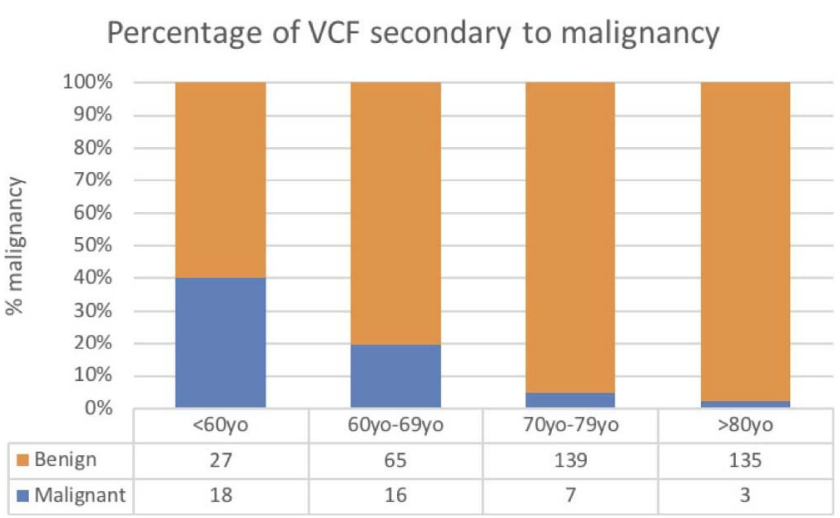

Figure 2. Percentage of vertebral compression fractures (VCFs) secondary to malignancy for patients who underwent percutaneous vertebral augmentation procedures (PVAPs). The $<60$ years age group had the largest percentage of VCF secondary to malignancy, of $40 \%$, followed by $19.8 \%, 4.8 \%$, and $2.2 \%$ for 60 to 69,70 to 79 , and $>80$ years age groups, respectively.

\section{DISCUSSION}

The incidence of malignancy in our study population was $11 \%$ (45 of 410 ). The rate of unsuspected malignancy in our study was $0.24 \%$ ( 1 of 410). This is significantly lower than previous studies. ${ }^{7,12,13}$ The single case of unsuspected malignancy picked up on biopsy was that of multiple myeloma, and this patient had positive monoclonal band on preoperative blood investigations. Thus, our study showed that MRI together with a myeloma panel was able to rule out all malignancies prior to the biopsy.

Although VCFs in this study population were spread out across various vertebral levels, most occurred at the thoracolumbar junction. Nearly half of all VCFs studied occurred at T12 or L1 (47.2\%) because of the higher biomechanical stresses at the junction. ${ }^{14,15}$ Although primary malignancies can occur at any level, metastatic disease tends to occur at the thoracic region, $\mathrm{T} 4$ to $\mathrm{T} 11$, followed by the lumbar region. ${ }^{16-18}$

The accuracy of MRI in diagnosing benign and malignant VCF has been reported to be as high as 94\%. ${ }^{10,19-21}$ The results of our study show that MRI can pick up $97.8 \%$ (44 of 45 positive biopsies) of malignant lesions, which is higher than previous reported studies. Moreover, this study revealed that when MRI scans were done in conjunction with a

Table 3. Odds ratios and $95 \%$ confidence intervals for different age ranges.

\begin{tabular}{lccc}
\hline Age Range, $\mathbf{y}$ & Sample Size, $\mathbf{n}$ & Odds Ratio & $\mathbf{9 5 \%}$ Confidence Interval \\
\hline$<60$ & 45 & 28.333 & $7.760-103.451$ \\
$60-70$ & 81 & 12.593 & $4.765-33.280$ \\
$70-80$ & 146 & 2.519 & $1.112-5.704$ \\
$>80$ & 138 & Control & Control \\
\hline
\end{tabular}
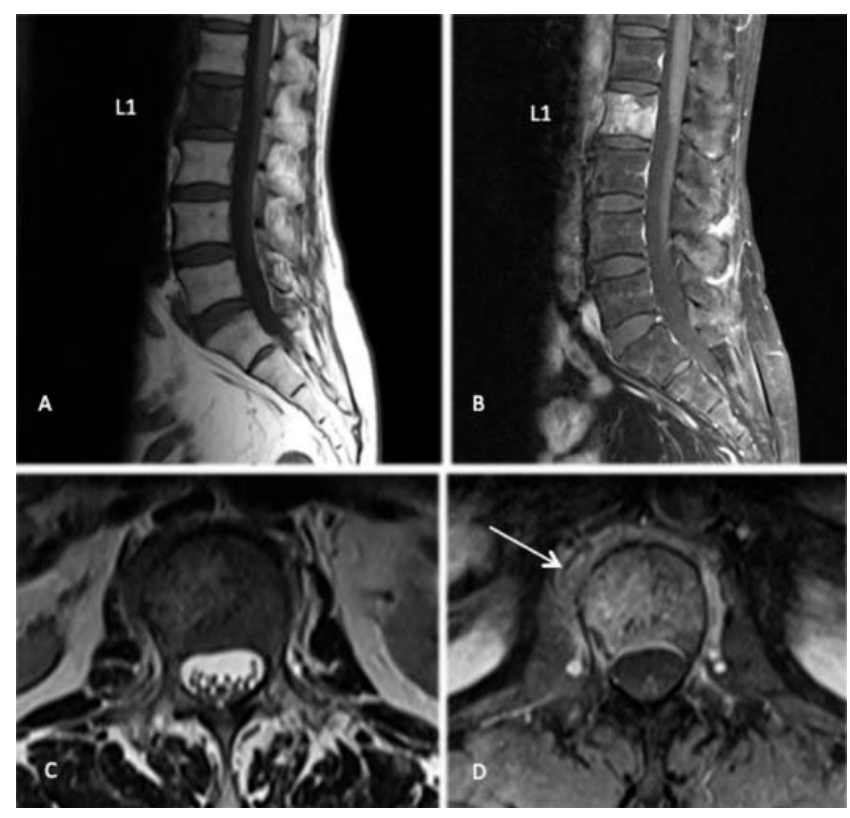

Figure 3. (A) Sagittal T1W; (B) sagittal T1FS + contrast; (C) axial T2W at L1 level; (D) axial T1FS + contrast at L1 level. There is a T1 hypointense lesion demonstrating contrast enhancement involving almost the entire L1 vertebral body in a patient with background breast cancer. Furthermore there is enhancement of the paravertebral soft tissue at this level suspicious for extraosseous component (indicated with an arrow). Note L5 is sacralised.

myeloma panel, no malignancies were missed. Sample MRI images of VCF due to pathologic and nonpathologic causes are shown in Figures 3 and 4.

In this study, a single patient was found to have multiple myeloma on histologic studies, whereas
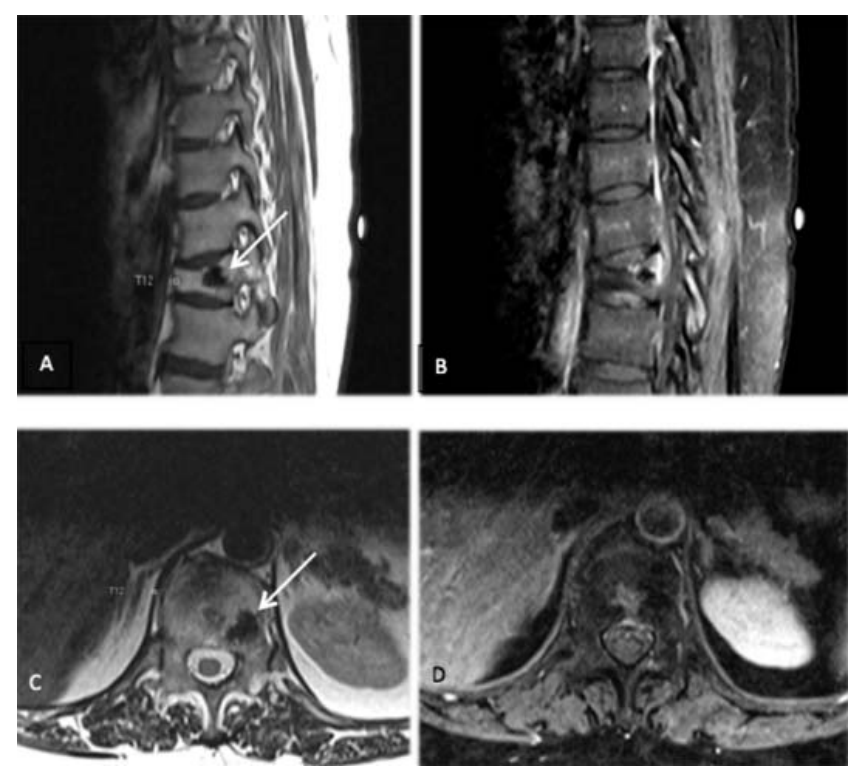

Figure 4. (A) Sagittal T1W; (B) T1W FS with contrast; (C) axial T2W at T12; (D) axial T1 FS with contrast at T12. MRI thoracic spine of a patient with nonpathological grade 3 compression fracture of T12 vertebra status-post vertebroplasty (low signal indicated by arrow). Compare the relatively normal T1 bone marrow signal and lack of contrast enhancement and spinal canal involvement with prior figure of a patient with spinal metastasis. 


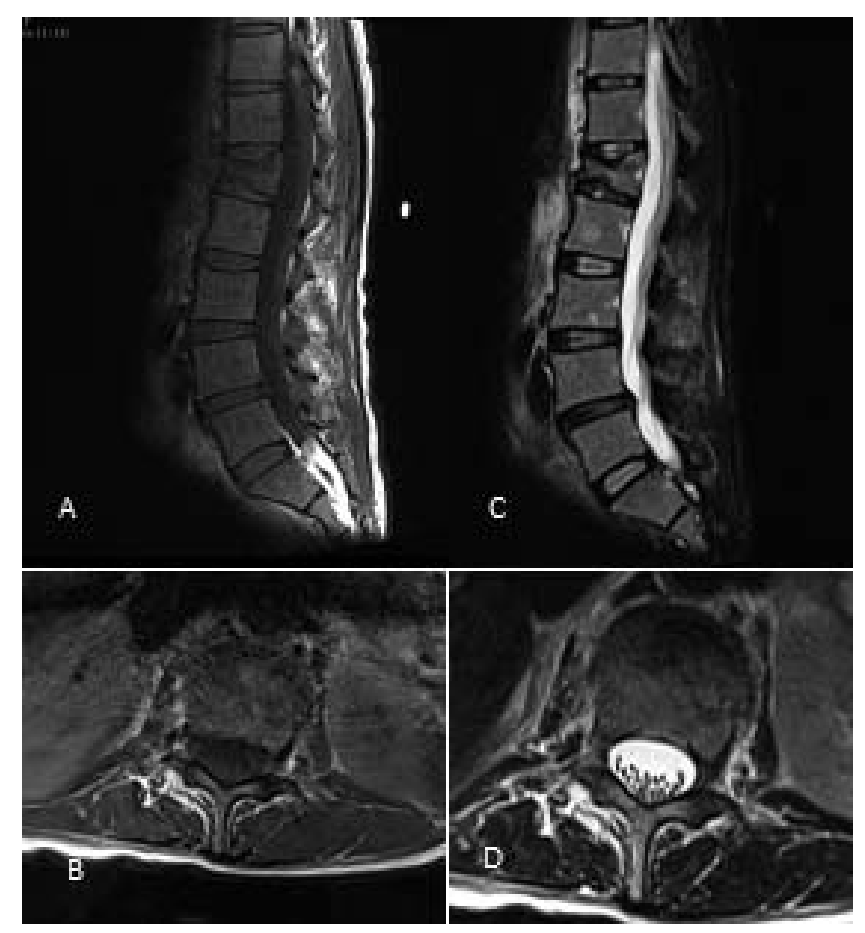

Figure 5. (A) Sagittal T1W; (B) axial T1W at L1; (C) sagittal STIR sequence; (D) axial T2W at L1. MRI lumbar spine of patient with unsuspected malignancy that showed no suspicious features of malignancy.

preoperative MRI images showed no features of malignancy. This patient was a 60 -year-old woman who presented with a history of dull low back pain of a few months' duration that was associated with fatigue and weight loss. As shown in Figure 5, the classical "salt and pepper" pattern of marrow infiltration was not demonstrated in the MRI images of this patient. ${ }^{22}$ Instead, a more uncommon pattern of diffuse osteopenia resulting in compression fracture was seen. Nevertheless, plain radiographs of the skull of this patient demonstrated multiple small lucent lesions suspicious of multiple myeloma.

Although it is highly reassuring that MRI scans and blood investigations appear to be able to pick up malignant causes of $\mathrm{VCF}$, it is often difficult to tell the etiology of the malignancy. Biopsy would be beneficial for patients presenting with VCF due to primary or secondary malignancy without any history of malignancy because MRI can only detect the presence of malignant features but not the etiology of the malignancy. Furthermore, histologic diagnosis remains essential in guiding further treatment of the identified malignancy. With the biopsy done concurrent with PVAP, not causing additional trauma, further biopsies may be avoided, thus reducing additional operative risks and patient discomfort. The rate of vertebral fractures due to malignancy is as high as $10 \%$, and because biopsy during PVAP does not cause additional trauma and can determine the etiology of malignancy, we recommend biopsy of all vertebral fractures during a PVAP. The role of the MRI may be even more invaluable in symptomatic VCF despite trial of nonoperative treatment and refusing any interventional procedures such as biopsy. Appropriate blood investigation together with an MRI may be able to pick up underlying pathologic fractures secondary to malignancy, notably multiple myeloma.

This study also found that younger patients (younger than 60 years) with VCF were approximately 30 times more likely to present with malignant VCF compared with patients older than 80 years. We postulate that this may be due to the increased number of osteoporotic patients in the older age group. As patients get older from 60 years onwards, the percentage of pathologic VCF decreases. Therefore, a high index of suspicion for malignancy should be maintained in young patients presenting with VCF.

The strength of this study lies in the relatively large patient population size. The authors acknowledge the limitation of the retrospective nature of the study. There is also likely to be some degree of reporting bias to the study because 41 of the 44 patients with malignancy had a known primary. We also note that of all patients who underwent PVAP, 326 did not have specimens sent for biopsy because these patients already had features suggestive of osteoporotic VCFs based on preoperative MRIs. We acknowledge that these 326 patients are a potential source of malignancies missed on preoperative MRIs.

\section{CONCLUSION}

This study shows that $10 \%$ of VCFs undergoing PVAP are pathologic for malignancy. The rate of unsuspected malignancy is $0.24 \%$ ( 1 of 410 biopsies). Almost $98 \%$ of patients with malignancy (44 of 45 patients) can be successfully identified with a preoperative MRI. This is significantly higher than previously reported. The addition of a myeloma panel to the MRI scan identified all patients with malignancies prior to PVAP in our study. Identification of malignancy prior to the procedure may have subsequent management consequences. This study recommends an MRI and a myeloma panel 
for all patients who have persistent pain from a VCF. This is especially important for patients who do not undergo an operative procedure where there is no opportunity for a biopsy to be taken. For patients who undergo a PVAP, routine biopsy should be performed. In addition, clinicians should adopt a high index of suspicion for malignancy in younger patients (younger than 60 years) with VCF.

\section{ACKNOWLEDGMENTS}

The devices/drugs in the present study are approved by the US Food and Drug Administration or approved the corresponding national agency for the indications examined.

\section{REFERENCES}

1. Greenberg MS. Handbook of Neurosurgery. 6th ed. New York, NY: Thieme Publishing Group; 2005.

2. Nuti R, Caffarelli C, Guglielmi G, Gennari L, Gonnelli S. Undiagnosed vertebral fractures influence quality of life in postmenopausal women with reduced ultrasound parameters. Clin Orthop Relat Res. 2014;472(7):2254-2261.

3. McGraw JK, Lippert JA, Minkus KD, Rami PM, Davis TM, Budzik RF. Prospective evaluation of pain relief in 100 patients undergoing percutaneous vertebroplasty: results and follow-up. J Vasc Interv Radiol. 2002;13(9, pt 1):883-886.

4. Voormolen M, Mali W, Lohle P, et al. Percutaneous vertebroplasty compared with optimal pain medication treatment: short-term clinical outcome of patients with subacute or chronic painful osteoporotic vertebral compression fractures: the VERTOS study. AJNR Am J Neuroradiol. 2007;28(3):555560 .

5. Zoarski GH, Stallmeyer MB, Obuchowski A. Percutaneous vertebroplasty: A to Z. Tech Vasc Interv Radiol. 2002;5(4):223-238.

6. Armsen N, Boszczyk B. Vertebro-/kyphoplasty history, development, results. Eur J Trauma. 2005;31(5):433-441.

7. Muijs SPJ, Akkermans PA, Van Erkel AR, Dijkstra SD. The value of routinely performing a bone biopsy during percutaneous vertebroplasty in treatment of osteoporotic vertebral compression fractures. Spine (Phila Pa 1976). 2009;34(22):2395-2399.

8. Pneumaticos SG, Chatziioannou SN, Savvidou C, Pilichou A, Rontogianni D, Korres DS. Routine needle biopsy during vertebral augmentation procedures. Is it necessary? Eur Spine J 2010;19(11):1894-1898.

9. Togawa D, Lieberman IH, Bauer TW, Reinhardt MK Kayanja MM. Histological evaluation of biopsies obtained from vertebral compression fractures: unsuspected myeloma and osteomalacia. Spine (Phila Pa 1976). 2005;30(7):781-786.

10. An HS, Andreshak TG, Nguyen C, Williams A, Daniels D. Can we distinguish between benign versus malignant compression fractures of the spine by magnetic resonance imaging? Spine (Phila Pa 1976). 1995;20(16):1776-1782.
11. Chou KN, Lin BJ, Chien LY, Tsai WC, Ma HI, Hueng DY. Simple transpedicular vertebral biopsy for diagnosis of malignancy in vertebral compression fracture. Neurol India. 2013;61(6):587.

12. Hansen EJ, Simony A, Carreon L, Andersen MO. Rate of unsuspected malignancy in patients with vertebral compression fracture undergoing percutaneous vertebroplasty. Spine (Phila Pa 1976). 2016;41(6):549-552.

13. Mukherjee S, Thakur B, Bhagawati D, et al. Utility of routine biopsy at vertebroplasty in the management of vertebral compression fractures: a tertiary center experience: clinical article. J Neurosurg Spine. 2014;21(5):687-697.

14. Daffner R. Imaging of thoracic and lumbar vertebral fractures. In: Floman Y, Farcy JPC, Argenson C, eds. Thoracolumbar Spine Fractures. New York, NY: Raven Press; 1993:69.

15. Knop C, Blauth M, Bühren V, et al. Surgical treatment of injuries of the thoracolumbar transition, 1: epidemiology [in German]. Unfallchirurg 1999;102(12):924-935.

16. Gerszten PC, Welch WC. Current surgical management of metastatic spinal disease. Oncology (Willison Park). 2000;14(7):1013-1044.

17. Klimo P, Schmidt MH. Surgical management of spinal metastases. Oncologist. 2004;9(2):188-196.

18. Cole JS, Patchell RA. Metastatic epidural spinal cord compression. Lancet Neurol. 2008;7(5):459-466.

19. Rupp RE, Ebraheim NA, Commbs RJ. Magnetic resonance imaging defferentiation of compression spine fractures or vertebral lesions caused by osteoporosis or tumor. Spine (Phila Pa 1976). 1995;20(23)2499-2503.

20. Hamaoka T, Madewell JE, Podoloff DA, Podoloff DA, Hortobagyi GN, Ueno NT. Bone imaging in metastatic breast cancer. J Clin Oncol. 2004;22(14):2942-2953.

21. Moulopoulos LA, Yoshimitsu K, Johnston DA, Leeds NE, Libshitz HI. MR prediction of benign and malignant vertebral compression fractures. J Magn Reson Imaging. 1996;6(4):667-674.

22. Delorme S, Baur-Melnyk A. Imaging in multiple myeloma. Eur J Radiol. 2009;70(3):401-408.

Disclosures and COI: No funds were received by any authors in preparation of this manuscript.

Corresponding Author: Chew Zhihong, Department of Orthopaedic Surgery, Singapore General Hospital, The Academia, 20 College Road, Singapore 169856. Phone: 85117982; Email: zhouzhihong0@gmail.com.

Published 31 October 2019

This manuscript is generously published free of charge by ISASS, the International Society for the Advancement of Spine Surgery. Copyright (C) 2019 ISASS. To see more or order reprints or permissions, see http://ijssurgery.com. 